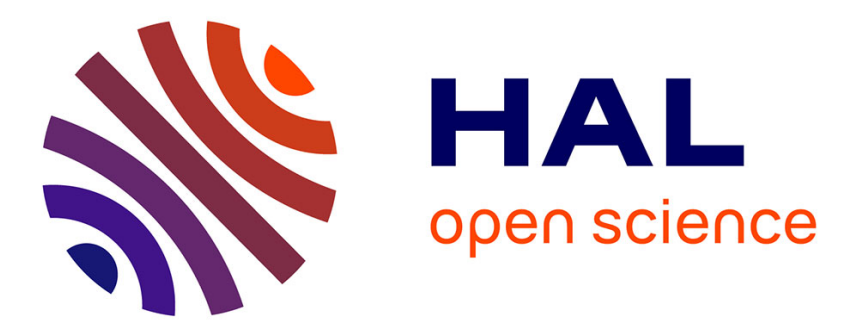

\title{
Dependence of turbulent advection on the Lagrangian correlation time
}

\author{
Wouter J.T. Bos, Le Fang
}

\section{To cite this version:}

Wouter J.T. Bos, Le Fang. Dependence of turbulent advection on the Lagrangian correlation time. Physical Review E: Statistical, Nonlinear, and Soft Matter Physics, 2015, 91, pp.043020. 10.1103/PhysRevE.91.043020 . hal-01298328

\section{HAL Id: hal-01298328 \\ https://hal.science/hal-01298328}

Submitted on 8 Apr 2016

HAL is a multi-disciplinary open access archive for the deposit and dissemination of scientific research documents, whether they are published or not. The documents may come from teaching and research institutions in France or abroad, or from public or private research centers.
L'archive ouverte pluridisciplinaire HAL, est destinée au dépôt et à la diffusion de documents scientifiques de niveau recherche, publiés ou non, émanant des établissements d'enseignement et de recherche français ou étrangers, des laboratoires publics ou privés. 


\title{
Dependence of turbulent advection on the Lagrangian correlation time
}

\author{
Wouter J. T. Bos ${ }^{1}$ and Le Fang ${ }^{2}$ \\ ${ }^{1}$ LMFA, CNRS, Ecole Centrale de Lyon, Université de Lyon, 69134 Ecully, France \\ ${ }^{2}$ Ecole Centrale de Pékin, Beihang University, 100191 Beijing, China
}

(Received 12 November 2014; revised manuscript received 13 February 2015; published 28 April 2015)

\begin{abstract}
In turbulent scalar mixing, starting from random initial conditions, the root-mean-square advection term rapidly drops as the flow and the scalar field organize. We show first analytically, for the simplified case of a blob in shear flow with a finite correlation time, how the advection term is reduced compared to a randomly aligned scalar structure. This picture is then generalized to turbulent mixing. These examples show that the rapid depletion of advection depends on the lifetime of turbulent structures, compared to the local straining time scale. A turbulence closure is used to show that the Lagrangian correlation time indeed determines the deviation from Gaussian behavior. In particular it is shown that in the inertial range the depletion mechanism is self-similar, since a constant ratio is observed between the advection spectrum and its Gaussian equivalent. Finally, direct numerical simulation shows that in the limit of an infinite correlation time of the turbulent eddies, corresponding to a frozen velocity field, the mean-square advection tends to a zero fraction of its Gaussian estimate.
\end{abstract}

DOI: 10.1103/PhysRevE.91.043020

PACS number(s): 47.27.eb, 47.27.Gs

\section{INTRODUCTION}

A salient feature of hydrodynamic turbulence is its tendency to self-organize into coherent vortical structures. How exactly this self-organization takes place, and to what kind of flow pattern a turbulent velocity field will evolve, depends on the interplay of pressure, viscous dissipation, nonlinear advection, and forcing. Despite a century of efforts, no general principle explaining the self-organization of three-dimensional turbulence has been identified yet. An interesting suggestion of such a principle is the premise that turbulence will tend to organize towards a force-free state, where vorticity and velocity are aligned [1], and since the time this was suggested, a large number of investigations have focused on the detailed alignment properties of turbulence (see for instance [2]). In Ref. [3] a weak tendency of velocity-vorticity alignment was indeed observed in turbulent flows, but not enough to explain in detail the weakening of the nonlinearity and its related possible appearance of a force-free state. It was therefore suggested that this alignment might be just one manifestation of a more general principle.

Indeed the work of Kraichnan and Panda [4] illustrated that not only turbulence organizes towards a state in which the variance of the fluctuations of the nonlinear term are weaker than in a Gaussian field with the same energy distribution, but that this effect is also observed in model systems where no geometrical relation between vorticity and velocity exists. Subsequent work by Herring and Métais [5] and Bos et al. [6] further illustrated the possible generality of this self-organization principle by showing that a similar effect is also observed in the mixing of a passive scalar. In that case the mean-square advection term is reduced compared to its Gaussian estimate. Most of the considerations in these works [4-6] were purely statistical in nature, in the sense that no direct link was made to the underlying flow topology leading to the suppression of the strength of the nonlinearity. In contrast, other investigations, focusing on alignment properties, in particular the alignment of scalar gradients or material line elements with the eigenvectors of the velocity gradient tensor, have received considerable interest (see [7-10] and references therein), not so much in relation to the possible existence of a force-free state, but to describe the turbulent topology in general.

In the present investigation we will reconsider the case of the turbulent passive scalar, more tractable than the velocity case, to identify direct links between the local flow evolution, Lagrangian coherence, and the generation of non-Gaussian statistics. Also we will present a statistical multiscale analysis to investigate the underlying mechanisms as a function of length scale. In particular we will use the fact that in turbulence closures one can vary the ratio between strain rate and correlation time in a simple way. The continuous variation of this ratio allows one to show the direct link between the strength of the mean-square advection term and the Lagrangian correlation time.

The case we are interested in is an isotropic turbulent velocity field $\boldsymbol{u}(\boldsymbol{x}, t)$, advecting an isotropic passive scalar $\theta(\boldsymbol{x}, t)$ with diffusivity $D$,

$$
\partial_{t} \theta-D \Delta \theta=-\boldsymbol{u} \cdot \nabla \theta .
$$

It was observed [5,6] that the efficiency of the turbulent advection, compared to a Gaussian field, satisfies

$$
\frac{\left\langle(\boldsymbol{u} \cdot \nabla \theta)^{2}\right\rangle_{\text {turbulence }}}{\left\langle(\boldsymbol{u} \cdot \nabla \theta)^{2}\right\rangle_{\text {Gaussian }}}<1,
$$

where the brackets denote an ensemble average. The quantities here are the mean-square value of the advection term in a turbulent flow mixing a passive scalar compared to the same quantity in a Gaussian reference flow. The latter consists of random independent Fourier modes with the same kinetic energy spectrum as the turbulent flow, and the scalar fluctuation field also consists of independent modes having the same scalar variance spectrum as the turbulently advected scalar field. Since our Gaussian reference fields are defined to have the same energy distribution as the turbulent and scalar fields, the values of $\left\langle|\boldsymbol{u}|^{2}\right\rangle$ and $\left\langle|\nabla \theta|^{2}\right\rangle$ in the turbulent and Gaussian fields are equal. Moreover, because

$$
(\boldsymbol{u} \cdot \nabla \theta)^{2}=\|\boldsymbol{u}\|^{2}\|\nabla \theta\|^{2} \cos ^{2}(\alpha),
$$


with $-\pi \leqslant \alpha<\pi$, the angle between $\boldsymbol{u}$ and $\nabla \theta$, one possibility to observe a state in which Eq. (2) holds, is to have a decreased probability to have alignment between $\boldsymbol{u}$ and $\nabla \theta$. It is this possibility which is explored in the next section. In particular, we will discuss how a reduced mean-square value of the cosine of $\alpha$ can be related to the correlation time of eddies in the turbulent flow. The quantity that we investigate is thus $\left\langle\cos ^{2}(\alpha)\right\rangle$. For a random orientation, this quantity is equal to $1 / 3$ in three dimensions, and $1 / 2$ in two dimensions.

In the following section we consider a blob in shear flow, which is the simplest nontrivial example which can be used to get a grip on the geometrical description of the depletion of advection. Section III generalizes the ideas to turbulent flows and illustrates using closure theory how the correlation time of structures is related to the generation of non-Gaussian behavior. Section IV illustrates how the advection is depleted in the case of infinite correlation time, i.e., in a frozen velocity field. In Sec. V we will discuss the link between the depletion of advection and its geometrical interpretation, with the depletion of nonlinearity in the Navier-Stokes equations, and several related issues.

\section{A BLOB IN CONSTANT SHEAR FLOW}

To illustrate a mechanism which leads to the depletion of advection, we consider a circular blob of size $l_{0}$ of scalar $\Theta$ in a uniform shear flow,

$$
\boldsymbol{u}=S x \boldsymbol{e}_{y},
$$

where $S=\partial u_{y} / \partial x$ is constant, as sketched in Fig. 1(a). In this example we consider for simplicity the two-dimensional case, but all the ideas can be straightforwardly transposed to three dimensions. After some time the blob will be stretched and rotated as sketched in Fig. 1(b). As the blob gets stretched, the gradients of the scalar will get enhanced until diffusion sets in. We will assume a vanishingly small diffusion in the following. The total amount of scalar is then conserved so that when the blob is stretched, its surface $\pi l_{0}^{2} / 4$ will remain roughly constant (in three dimensions we have a volume which remains unchanged, with the dimension of the blob in the cross-stream direction remaining unchanged). We will denote by $l(t)$ the size of the blob in the direction of maximum stretching. In the direction of strongest compression the typical size of the blob is $d(t)$. We will thus have an elliptical scalar blob for $t>0$.
Since its surface remains constant, we have

$$
l(t) d(t) \sim l_{0}^{2}=\text { constant. }
$$

We can now estimate the gradients. In the direction $\boldsymbol{e}_{\perp}$ the strength of the scalar gradient $\nabla_{\perp} \theta$ will be

$$
\left\|\nabla_{\perp} \theta\right\| \sim \Theta / d(t)
$$

The parallel gradient will be lower, of order $\Theta / l(t)$, since $l>d$. The important thing here is that, evidently, the dominant scalar gradients are perpendicular to the direction in which the blob is stretched. Their values are not important in our argument.

While the stretched blob aligns more and more with the streamlines, the dominant scalar gradient gets increasingly perpendicular to them. We can compute the angle $\alpha(t)$ in Fig. 1(c) between the direction of the dominant scalar gradient and the velocity. In our shear flow, the blob will get sheared as the positions of material points $\boldsymbol{x}_{1}$ and $\boldsymbol{x}_{2}$ get separated. The distance between these points is $l(t)=\left\|\boldsymbol{x}_{1}-\boldsymbol{x}_{2}\right\|$. Since the shear is uniform, we have $\dot{x_{1}}=\frac{1}{2} S l_{0} t \boldsymbol{e}_{y}=-\dot{\boldsymbol{x}_{2}}$. Therefore, the length of the blob evolves to a good approximation as

$$
l(t)=l_{0} \sqrt{1+S^{2} t^{2}} .
$$

The angle in Fig. 1(c) is thus

$$
\cos \alpha=l_{0} / l=\frac{1}{\sqrt{1+S^{2} t^{2}}} .
$$

Averaged over a time interval, we have for this simple case

$$
\begin{aligned}
\left\langle\cos ^{2} \alpha\right\rangle_{T} & \equiv \frac{1}{T} \int_{0}^{T} \cos ^{2} \alpha d t, \\
& =\frac{\arctan (S T)}{S T} .
\end{aligned}
$$

This quantity will thus tend to zero for large values of $S T$, which we understand intuitively from the sketch in Fig. 1, since the blob aligns increasingly in time with the streamlines.

We will now consider the case where the shear flow changes its direction randomly every time interval $T$. Reconsidering the same example as above, we have now

$$
\cos \alpha=\frac{1}{\left.\sqrt{1+\left[\tan \left(\alpha_{0}\right)+t S\right.}\right]^{2}},
$$

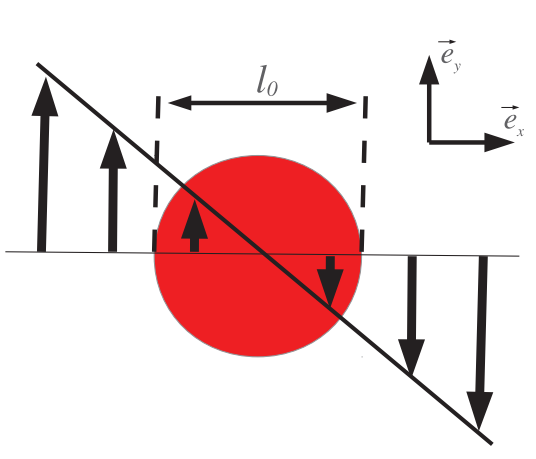

(a)

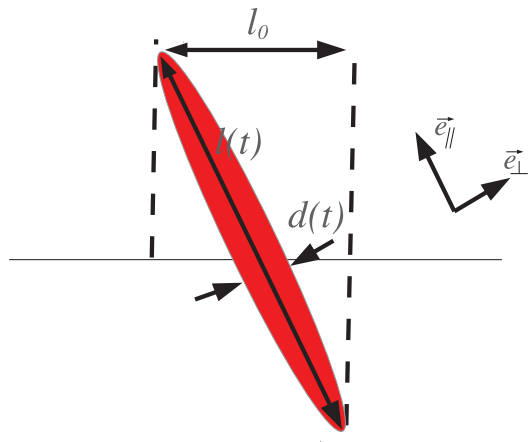

(b)

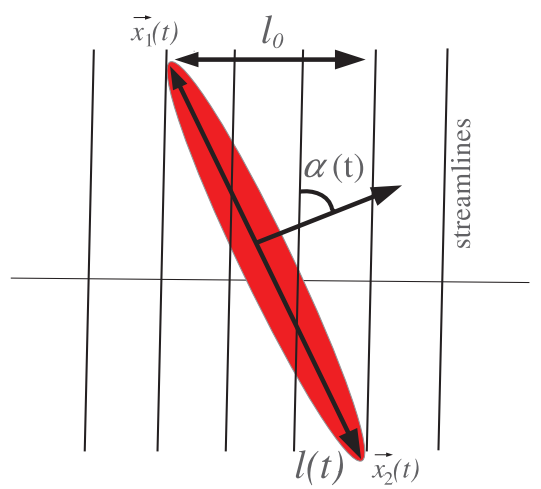

(c)

FIG. 1. (Color online) Stretching a blob in a constant shear flow. 


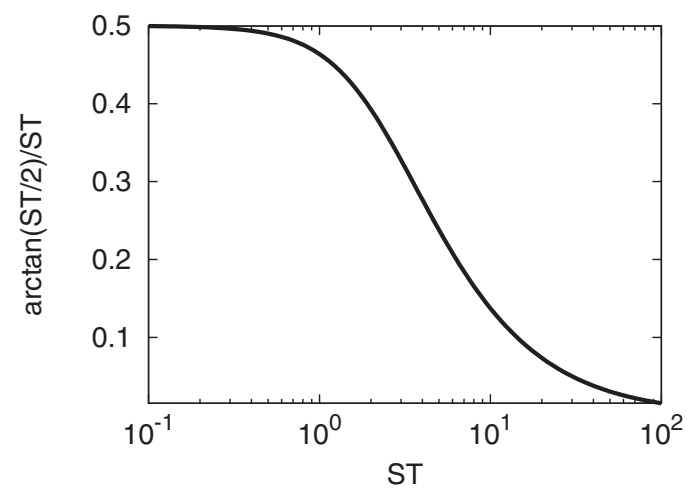

FIG. 2. Dependence of the mean-square cosine of the angle between the velocity field and the dominant gradient in a randomly oriented blob of the scalar as a function of the correlation time.

where $-\pi / 2<\alpha_{0}<\pi / 2$ is the angle between the flow and the direction of the dominant scalar gradient at the beginning of the interval. Averaged over a time interval $T$, we obtain for the square of the cosine

$$
\left\langle\cos ^{2} \alpha\left(\alpha_{0}\right)\right\rangle_{T}=\frac{\arctan \left(\tan \alpha_{0}+S T\right)-\alpha_{0}}{S T} .
$$

We consider that the orientation of the shear is independent of the orientation of our stretched filament. Every value of $\alpha_{0}$ is therefore equally probable and the average value of $\left\langle\cos ^{2} \alpha\left(\alpha_{0}\right)\right\rangle_{T}$ over an infinite number of realizations is then

$$
\begin{aligned}
\left\langle\cos ^{2}(\alpha)\right\rangle & =\frac{1}{\pi} \int_{-\pi / 2}^{\pi / 2}\left\langle\cos ^{2} \alpha\left(\alpha_{0}\right)\right\rangle_{T} d \alpha_{0} \\
& =\frac{\arctan (S T / 2)}{S T} .
\end{aligned}
$$

We show expression (12) in Fig. 2. It is observed that for $S T \downarrow 0$ the value of $\left\langle\cos ^{2}(\alpha)\right\rangle$ tends to $1 / 2$. This corresponds to the mean-square value of the cosine of the angle between two randomly orientated vectors in two dimensions. Indeed, if the stretched blob of the scalar is reoriented too rapidly for the dynamics to act, the angle will be randomly distributed. However, if the value $S T$ is taken towards infinity, the blob will over each interval $T$ be stretched and oriented towards the direction of the velocity. The value of $\left\langle\cos ^{2} \alpha\right\rangle$ drops from 0.5 to 0 for values of $S T>1$. It is shown that for values $S T<1$ the influence of stretching is smaller than $10 \%$ and for $S T>100$ the depletion is almost complete. Comparing expressions (9) and (12), we see that for $S T \downarrow 0$, the ratio is a factor of 2 . This factor is induced by the random orientation in the second case.

In reality the shear will not be constant over a time interval and then change direction. We could refine the present analysis by assuming, for instance, an exponentially decaying shear over each time interval. However, for such a case the analytical forms of the alignment statistics become very complicated. We will not attempt such a refinement of the above picture, but rather focus on a phenomenological multiscale extension of the present ideas to suggest how the above mechanism can be transposed to a turbulent flow.

In this section we considered the value of the mean-square cosine of the angle $\alpha$. We mention here that evolution equations for the alignment angle were derived in [11]. These equations are, however, not less complicated than the Navier-Stokes and scalar equations. A further analysis of those equations might prove a complementary approach to better understanding the link between depletion of advection and the local topology of the scalar and velocity field and is left for further research. In the following we will consider the mean-square advection compared to its value in a Gaussian field. The alignment mechanism sketched in the foregoing is one of the mechanisms which can explain the reduction of the value of the meansquare advection and we will show in the following that observations in both direct numerical simulation (DNS) and closure are consistent with this idea.

\section{ADVECTION BY TURBULENCE}

Let us try to transpose these ideas for the advection by pure shear to turbulent mixing. In a high Reynolds number three-dimensional turbulent flow, a blob will not be strained forever at the same rate and in the same orientation. The time over which the blob will be stretched is a function of the scale $1 / k$, and this time scale will vary as

$$
\tau(k) \sim \epsilon^{-1 / 3} k^{-2 / 3},
$$

with $\epsilon$ the dissipation rate of kinetic energy. We assumed here typical scaling consistent with Kolmogorov's 1941 phenomenological description of inertial range dynamics [12], where the energy spectrum $E(k)$ is approximately given by

$$
E(k) \sim \epsilon^{2 / 3} k^{-5 / 3},
$$

for $1 / k$ a scale much smaller than the integral scale and much larger than the dissipative scale. The strain rate at this scale can be estimated as the total cumulative strain of scales larger than $1 / k$ as

$$
S(k)=\sqrt{\int_{0}^{k} p^{2} E(p) d p .}
$$

In the inertial range, $S(k)$ will be

$$
S(k) \sim \epsilon^{1 / 3} k^{2 / 3},
$$

proportional to $\tau(k)^{-1}$. If the picture of a blob in a shear flow as described in the previous section can be transposed to a blob of scale $1 / k$ in the inertial range, and if we suppose that local (in scale) interactions are dominating the deformation of such a blob, the advection as compared to its Gaussian measure should be controlled by the ratio of the correlation time $\tau(k)$ to the shear time $S(k)^{-1}$. Since, using the estimates (13) and (16),

$$
S(k) \tau(k) \sim k^{0},
$$

we can expect that in the inertial range the depletion of advection will be scale independent.

In the following we will use a statistical turbulence closure to illustrate these ideas. This allows us to tune parameters that are not easily modified in experiments or in the DNS of turbulence. In particular, we will vary a parameter which determines the ratio between the shearing time $S(k)^{-1}$ and the Lagrangian correlation time $\tau(k)$. How this parameter appears in the present model will be explained now.

In closure approaches such as the eddy-damped quasinormal Markovian model (EDQNM) [13], a parameter $\lambda$ 


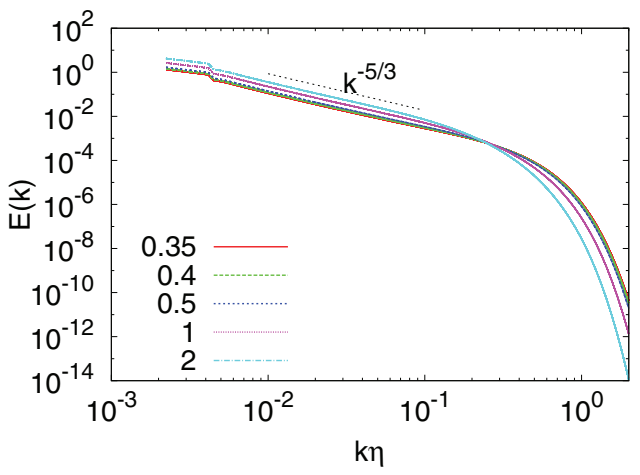

(a)

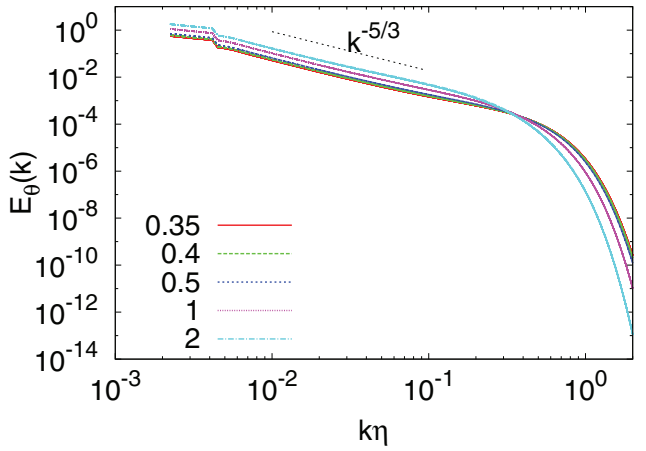

(b)

FIG. 3. (Color online) Wave-number spectra of the kinetic energy (a) and scalar variance (b) for different values of the parameter $\lambda$.

appears as an adjustable constant when the model is derived from Kraichnan's direct interaction approximation [14] by assuming exponentially decaying time correlations of the form

$$
\left\langle u_{i}(\boldsymbol{k}, t) u_{i}^{*}(\boldsymbol{k}, s)\right\rangle=\left\langle u_{i}(\boldsymbol{k}, t) u_{i}^{*}(\boldsymbol{k}, t)\right\rangle \exp \left(-\frac{|t-s|}{\tau(k)}\right),
$$

where $u_{i}(\boldsymbol{k}, t)$ is the Fourier transform of the velocity field $u_{i}(\boldsymbol{x}, t)$. Details on such a description can be found in [15]. In the EDQNM model the correlation time scale is subsequently modeled by

$$
\tau(k)^{-1}=\lambda \sqrt{\int_{0}^{k} p^{2} E(p) d p}+v k^{2} .
$$

In the inertial range, where the viscous term is negligibly small, we have, using Eq. (15),

$$
\lambda^{-1}=\tau(k) S(k),
$$

so that $\lambda^{-1}$ is exactly the ratio of the correlation time to the local shearing time as a function of scale. In this closure $\lambda$ is not fixed and is usually determined in order to obtain the correct Kolmogorov constant. Indeed, at high Reynolds numbers one finds that $\lambda \sim C_{k}^{3 / 2}$. The variation of $\lambda$ is thus directly related to a variation of the Kolmogorov constant, something which cannot be done experimentally, but which can help us to test the hypotheses proposed in the foregoing sections.

Following the ideas of Sec. II, where it was shown for a blob in shear flow that the mean square of the cosine of the angle $\alpha$ and thereby the mean-square advection is reduced compared to a randomly oriented blob for large $S T$, it can be expected that decreasing the value of $\lambda$ will increase the tendency to reduce the mean-square advection term. We have checked this using our recently derived closure theory for the mean-square advection term [6]. We have varied the values of $\lambda$ and $\lambda_{\theta}$ appearing in the closure of the velocity and passive scalar dynamics, respectively [Eq. (37) of Ref. [6]], between 0.35 and 2 . This changes the results for the statistics of the velocity field and the scalar field. The details of these simulations can be found in [6]. Both velocity and scalar are forced to obtain a steady state. The difference with respect to the simulations reported in [6] is that we have varied the model constants in order to check the above ideas on the relation between a scale-dependent Lagrangian correlation time and the reduction of the strength of the advection term. We have changed both constants simultaneously, keeping $\lambda=\lambda_{\theta}$, but we stress that in principle both constants could be changed independently.

In Fig. 3, we show the energy spectra and scalar spectra obtained in the statistically steady state. Clearly, the qualitative behavior of the spectra is not so strongly influenced, but the precise value of the Kolmogorov and Corrsin-Obukhov constants is modified when we change $\lambda$. This is shown in Fig. 4 where we present the results for the compensated spectra.

Figure 5(a) shows the mean-square advection spectrum $w_{\theta}(k)$ for different values of $\lambda$. The advection spectrum is defined through the relation

$$
\int w_{\theta}(k) d k=\left\langle(\boldsymbol{u} \cdot \nabla \theta)^{2}\right\rangle \equiv A .
$$

The mean-square advection spectra compared to their Gaussian estimate are shown in Fig. 5(b). The ratio in the inertial range is roughly constant for all values, as was predicted in expression (17), but for the smallest values of $\lambda$ the power-law behavior in the inertial range becomes less pronounced. This constant ratio in the inertial-convective range was already observed in [6] where we also presented results from DNS (using the data from Ref. [16]).

The total value of the mean square advection compared to its Gaussian value, given by

$$
\frac{A_{T}}{A_{G}}=\frac{\left\langle(\boldsymbol{u} \cdot \nabla \theta)^{2}\right\rangle}{\frac{1}{3}\left\langle|\boldsymbol{u}|^{2}\right\rangle\left\langle|\nabla \theta|^{2}\right\rangle}
$$

is clearly a decreasing function of $\lambda^{-1}$, as can be seen in Fig. 6 .

\section{DNS OF ADVECTION BY A FROZEN VELOCITY FIELD}

A further check of the above scenario is now presented using direct numerical simulation. We will artificially change the coherence time of the eddies to infinity. Indeed, we will consider here the advection of the scalar by a frozen turbulent field. In that case the strain at a scale $k, S(k)$, will remain unchanged. However, the coherence time $\tau(k)$ of an eddy will tend to infinity since the eddy does not evolve in time. The scalar is not frozen and is mixed by the frozen velocity field. In this case, in expression (20) the strain rate will not change, but the time scale on which this strain will act on the scalar will be proportional to the time $t$. At long time, we will therefore have that the product $S(k) \tau(k)$ will increase proportional to $t$ in the case of the advection by a frozen field, whereas in an 


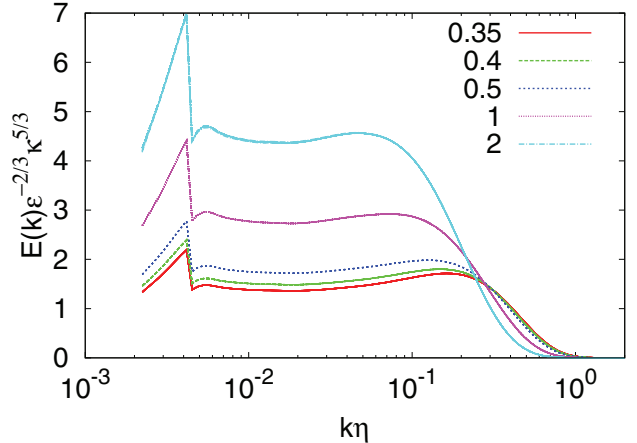

(a)

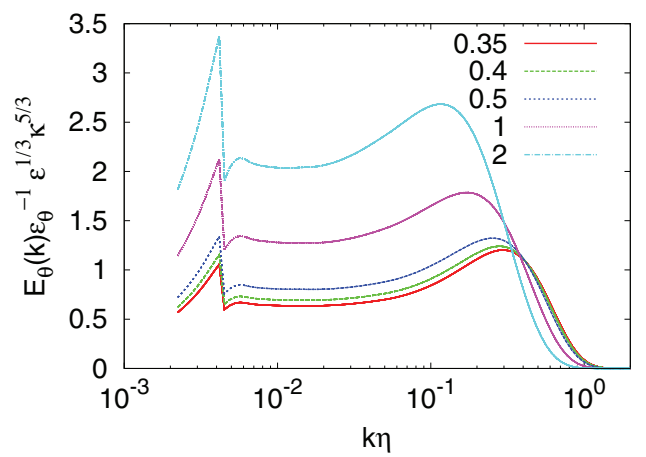

(b)

FIG. 4. (Color online) Compensated plots of the wave-number spectra of the kinetic energy (a) and scalar variance (b) for different values of the parameter $\lambda$.

inertial range this product is expected to be roughly constant in a freely evolving turbulent velocity field. The ratio of the mean-square advection to its Gaussian estimate is therefore expected to decrease more at long times in the frozen velocity case than in the freely evolving velocity case.

This will be checked in DNS in the following. We note here that similar simulations were presented in the work of Yang et al. [11], where the topology of Lagrangian scalar structures was investigated in both turbulence and a frozen velocity field. Their investigation of the evolution of the mean-square cosine of the angle of alignment between velocity and scalar gradient gives roughly the same results as the results we will present here for the mean-square advection term. This is consistent with the idea that for the case of the passive scalar, the depletion of advection is linked to the tendency of the scalar gradient to become perpendicular to the local velocity field.

We perform computations using a standard pseudospectral code using $128^{3}$ mesh points. This relatively low resolution is enough for our purposes. The initial conditions consist of a solenoidal field of Gaussian random numbers that yield the energy spectrum

$$
E(k)=B k^{4} \exp \left[-2\left(k / k_{0}\right)^{2}\right]
$$

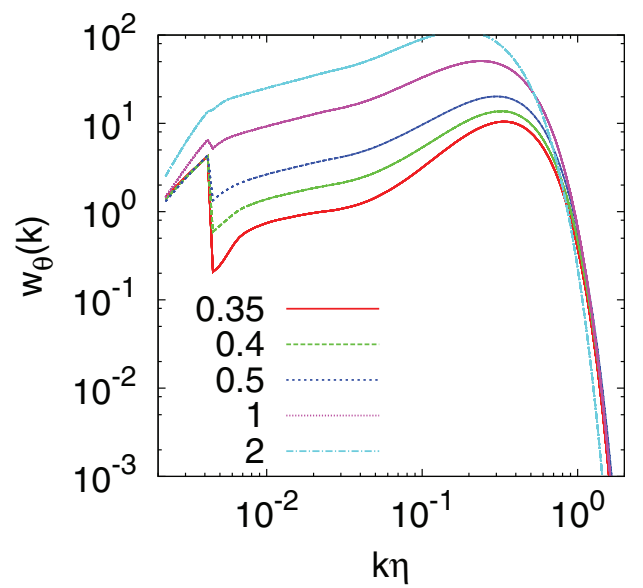

(a) with $k_{0}$ is 5 and $B$ a constant such that $\int E(k) d k=1$. The scalar field is chosen to have the same initial spectrum $E_{\theta}(k)$, but consists of an independent realization of random numbers. The value of the viscosity is chosen to be $v=0.012$, yielding a Taylor microscale Reynolds number $\operatorname{Re}_{\lambda}=50$ at $t=0$. The Prandtl number is equal to unity. The time value is normalized by the turn-over time at $t=0$. No forcing is added to the equations and the velocity and scalar are thus freely decaying.

In Fig. 7 we can observe, as in Kraichnan and Panda for the nonlinear term, that the ratio $A_{T} / A_{G}$ drops rapidly below unity. At a time instant where the flow has reduced the ratio to $A_{T} / A_{G} \approx 0.7$ we consider two different scenarios for further evolution. In the first situation, we let both the scalar and the velocity freely evolve. In the second situation we freeze the velocity field, but let the scalar field continue to evolve. This freezing of the velocity field will change the lifetime of the turbulent eddies. They will now have an infinite lifetime. The instantaneous strain rate experienced by the scalar field will not change since the velocity field does not change.

From Fig. 7 it is observed that whereas in the case of the freely decaying turbulence $A_{T} / A_{G}$ tends to a constant value, for the case of the frozen velocity field the ratio continues to decrease monotonically. We only show the development before

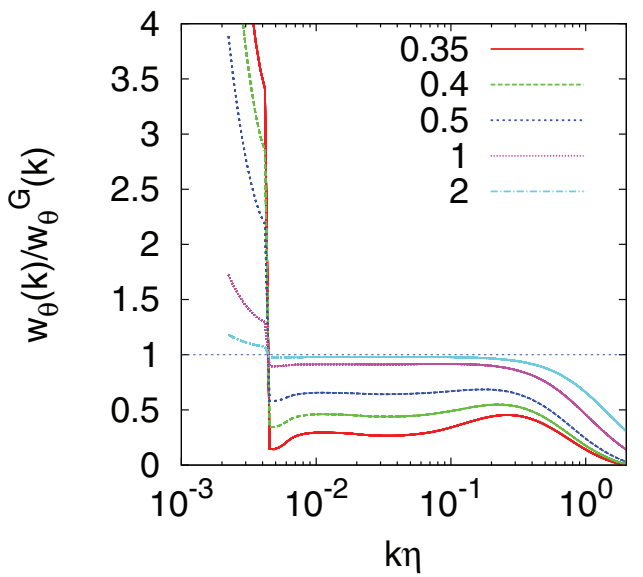

(b)

FIG. 5. (Color online) (a) Wave-number spectrum of the mean-square advection term. (b) The ratio of the spectra of the mean-square advection term to their Gaussian estimates. 


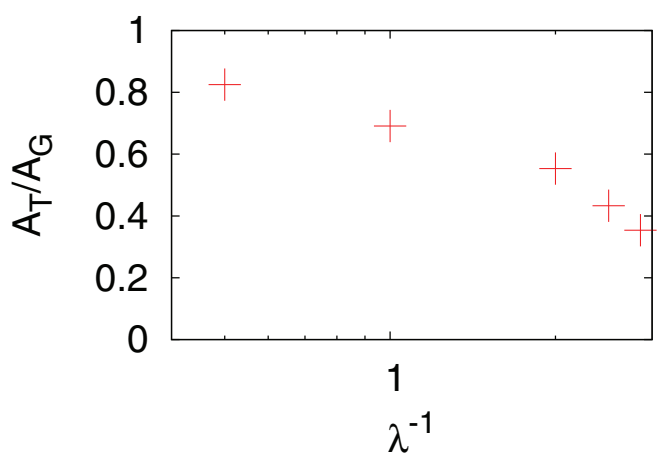

FIG. 6. (Color online) Mean-square advection term of the scalar equation in isotropic turbulence compared to its Gaussian value as a function of $\lambda^{-1}$.

$t=7$ in the figure. However, we have calculated these cases until $t=100$ and find that the value of $A_{T} / A_{G}$ continues to decrease in the case of a frozen velocity field.

\section{DISCUSSION}

\section{A. Advection in the Kraichnan model}

It has been known for some time that reorientation of a flow pattern in laminar mixing enhances its mixing efficiency [17]. It is not surprising that this is also the case in turbulence. What is interesting, however, is that this advection-reorientation principle is self-similar through the inertial range, as reflected by the constant ratio of the advection spectrum to its Gaussian estimate [see Fig. 5(b)]. We further mention here the implications for the advection strength in the Kraichnan model [18], which consists of the advection by a delta-correlated-in-time velocity field constituted by random solenoidal velocity field

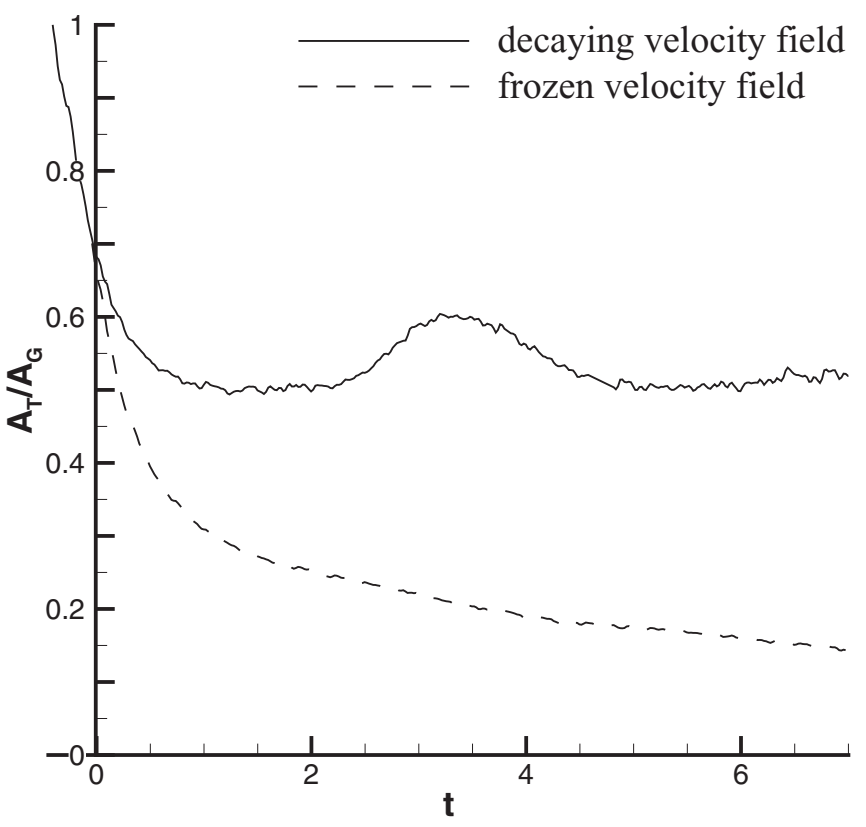

FIG. 7. Value of the mean-square advection normalized by its Gaussian value, for the advection of a passive scalar by both freely decaying turbulence and a frozen velocity field. with a prescribed energy distribution. Since the velocity field changes at every time instant, the angle will be randomly oriented at each time, and the angle between the scalar gradient and the velocity field will therefore also be so. We therefore expect in that case that the mean-square advection term will be equal to the Gaussian estimate. In the Kraichnan model the scalar structure functions display anomalous (i.e., nondimensional) scaling [19]. If our predictions are correct, this type of intermittency can thus be observed in a situation where the deviations from Gaussian behavior for the advection term vanish. The non-Gaussianity expressed by the depletion of advection seems thus not to be directly related to anomalous scaling in the inertial range.

\section{B. Depletion of advection and stretching of material line elements}

It is here perhaps interesting to mention the results presented in the work by Chen and Kraichnan [20]. They considered the stretching of a material line by a turbulent flow to investigate the influence of sweeping on the advection of a passive scalar. Initially, a line consisting of a large number of equidistant material points was advected in a random flow. The alignment of the line elements connecting the points with the local velocity field was tracked and statistical averages over a large number of realizations were taken. In their study also a comparison was made with a frozen velocity field. The line elements in the frozen field did not tend to completely align at long times, but the alignment tended to a constant mean absolute angle. They used this result to argue that the mean-square advection term and by analogy the mean-square nonlinear term in the Navier-Stokes equations could never reduce towards a zero fraction of their Gaussian estimates. This might seem at odds with the present results, but it is not so. The big difference in their approach is that they computed the angle between the velocity and the line segments that connected the two points that were initially equidistant. At long times these connecting points are evidently not equidistant anymore and they will describe a random motion. The orientation of connecting lines between two independent random points will be independent of the velocity field. This is not the case for material line elements. The statistics can completely change if the weight of the points is normalized with respect to the number of material points per unit length, thereby taking into account the stretching rate, as would be the case if we would truly follow the advection of a material line. This issue was recognized in work by Kida and Goto [21]. In our case this is an important issue, since line segments that are further stretched lead to stronger contributions to the scalar gradient. This difference explains the fact that in our frozen field the cosine of the angle between the velocity and the scalar gradient does not tend to a nonzero constant, but tends to zero.

\section{Influence of a large-scale forcing term}

A possibly important issue to further understand the depletion of nonlinearity is the influence of a large-scale forcing term. It is presently not clear how the alignment properties depend on the presence of a forcing term in order to maintain the flow in a statistically steady state. If the forcing rapidly 
changes the structure of the velocity field, the alignment might be negatively affected. The results could therefore strongly depend on the choice of the forcing term. In Sec. IV the velocity and scalar field decayed freely from a Gaussian initial condition. Kraichnan and Panda's investigation [4] of the depletion of nonlinearity was also carried out for decaying turbulence. For the depletion of advection, in Ref. [6] it was shown that in the presence of large-scale forcing the depletion of advection was still significant. It seems thus that for the scalar, the effect is robust, irrespective of the presence of a large-scale forcing. For the velocity field this is less sure. Indeed, closure seems to indicate that the depletion of nonlinearity is still significant [22]; however, in the forced direct numerical simulations of Ishihara et al. [23], the spectra of the mean-square nonlinear term seem to collapse with those of a Gaussian reference field. This issue needs further investigation.

\section{Depletion of vorticity advection}

It is of course tempting to transpose the scalar-advection picture from Sec. II to explain the depletion of the nonlinear term in turbulent flows, in particular since the spectral behavior of the mean-square nonlinearity behaves quite similar to the mean-square advection term [22]. If we replace the blob of the scalar in Fig. 1 by a blob of vorticity, stretching of the blob will reduce the alignment of the vorticity gradient with the velocity, thereby reducing the strength of the nonlinear vorticity advection term. Note that we focus on the dominant vorticity gradient, and not on the vorticity, which is shown to behave differently from the scalar gradient [7]. The relevant comparison in the present work is thus not between the scalar gradient and vorticity, but between the scalar gradient and the dominant vorticity gradient, which changes the main scope of the present work from the subject of most studies focusing on alignment. Evidently we should take into account that vorticity is not a passive quantity, and stretching the vorticity blob will necessarily induce a change in the velocity field, determined by the Biot-Savart integral relation. Our test case considering the advection by a frozen velocity field cannot be transposed easily to the case of the velocity either, since it is not possible to freeze the velocity field without freezing the vorticity. However, at short times it is probable that the mechanism sketched here for the scalar can be transposed to the vorticity and this can explain, at least in part, the tendency of velocity fields to have a nonlinearity that is small compared to that of a Gaussian field. This link between the depletion of scalar advection and the depletion of vorticity advection should be even closer for two-dimensional Navier-Stokes turbulence [24], since the dynamics of the vorticity is for this case also governed by an advection-diffusion equation.

A tempting conclusion of this work is that long-time correlations are the origin of non-Gaussian statistics, a statement which was proposed in the framework of Lagrangian intermittency $[25,26]$. We have substantiated that claim here in the case of scalar mixing, where it is clearly observed that both in the simple case of a scalar blob in shear flow and in the case of turbulent mixing, it is the ratio of the Lagrangian correlation time to the local shear time which sets the level of non-Gaussianity in the statistics. This non-Gaussianity does not seem to be linked to the anomalous scaling of spectra or structure functions.

\section{ACKNOWLEDGMENTS}

Robert Rubinstein is greatly acknowledged for his scientific input. Fang Le thanks the support of the National Natural Science Foundation in China (11202013, 51420105008) and the useful discussion with Jin Guo-Dong on Lagrangian particle deformation. We acknowledge interaction with Qu Bo on the deformation of a circular blob in shear flow, and discussion with Louis Gostiaux on the advection of material lines.
[1] R. Pelz, V. Yakhot, S. Orszag, L. Shtilman, and E. Levich, Velocity-vorticity patterns in turbulent flow, Phys. Rev. Lett. 54, 2505 (1985).

[2] A. Tsinober, An Informal Introduction to Turbulence (Kluwer, Dordrecht, 2001).

[3] M. M. Rogers and P. Moin, Helicity fluctuations in incompressible turbulent flows, Phys. Fluids 30, 2662 (1987).

[4] R. Kraichnan and R. Panda, Depression of nonlinearity in decaying isotropic turbulence, Phys. Fluids 31, 2395 (1988).

[5] J. Herring and O. Métais, Spectral transfer and bispectra for turbulence with passive scalars, J. Fluid Mech. 235, 103 (1992).

[6] W. Bos, R. Rubinstein, and L. Fang, Reduction of mean-square advection in turbulent passive scalar mixing, Phys. Fluids 24, 075104 (2012).

[7] W. T. Ashurst, A. R. Kerstein, R. M. Kerr, and C. H. Gibson, Alignment of vorticity and scalar gradient with strain rate in simulated Navier-Stokes turbulence, Phys. Fluids 30, 2343 (1987).

[8] S. Girimaji and S. Pope, Material-element deformation in isotropic turbulence, J. Fluid Mech. 220, 427 (1990).
[9] G. Brethouwer, J. C. R. Hunt, and F. T. M. Nieuwstadt, Microstructure and Lagrangian statistics of the scalar field with a mean gradient in isotropic turbulence, J. Fluid Mech. 474, 193 (2003).

[10] M. Gonzalez, Kinematic properties of passive scalar gradient predicted by a stochastic Lagrangian model, Phys. Fluids 21, 055104 (2009).

[11] Y. Yang, D. Pullin, and I. Bermejo-Moreno, Multi-scale geometric analysis of Lagrangian structures in isotropic turbulence, J. Fluid Mech. 654, 233 (2010).

[12] A. N. Kolmogorov, The local structure of turbulence in incompressible viscous fluid for very large Reynolds numbers, Dokl. Akad. Nauk. SSSR 30, 301 (1941).

[13] S. Orszag, Analytical theories of turbulence, J. Fluid Mech. 41, 363 (1970).

[14] R. Kraichnan, The structure of isotropic turbulence at very high Reynolds numbers, J. Fluid Mech. 5, 497 (1959).

[15] W. Bos and J.-P. Bertoglio, Lagrangian Markovianized field approximation for turbulence, J. Turbul. 14, 99 (2013).

[16] T. Watanabe and T. Gotoh, Statistics of a passive scalar in homogeneous turbulence, New J. Phys. 6, 40 (2004). 
[17] J. Ottino, The Kinematics of Mixing: Stretching, Chaos and Transport (Cambridge University, New York, 1989).

[18] R. H. Kraichnan, Small-scale structure of a scalar field convected by turbulence, Phys. Fluids 11, 945 (1968).

[19] R. Kraichnan, Anomalous scaling of a randomly advected passive scalar, Phys. Rev. Lett. 72, 1016 (1994).

[20] S. Chen and R. Kraichnan, Sweeping decorrelation in isotropic turbulence, Phys. Fluids A 1, 2019 (1989).

[21] S. Kida and S. Goto, Line statistics: Stretching rate of passive lines in turbulence, Phys. Fluids 14, 352 (2002).

[22] W. Bos and R. Rubinstein, On the strength of the nonlinearity in isotropic turbulence, J. Fluid Mech. 733, 158 (2013).
[23] T. Ishihara, Y. Kaneda, M. Yokokawa, K. Itakura, and A. Uno, Spectra of energy dissipation, enstrophy and pressure by high-resolution direct numerical simulations of turbulence in a periodic box, J. Phys. Soc. Jpn. 72, 983 (2003).

[24] A. Pushkarev and W. Bos, Depletion of nonlinearity in twodimensional turbulence, Phys. Fluids 26, 115102 (2014).

[25] N. Mordant, J. Delour, E. Leveque, A. Arneodo, and J.-F. Pinton, Long time correlations in Lagrangian dynamics: A key to intermittency in turbulence, Phys. Rev. Lett. 89, 254502 (2002).

[26] B. Kadoch, W. Bos, and K. Schneider, Origin of Lagrangian intermittency in drift-wave turbulence, Phys. Rev. Lett. 105, 145001 (2010). 